\title{
CRC algorithm in computer network communication
}

\author{
Baiming Liu \\ Department of Computer and Information Engineering \\ Beijing Science Technology Management College line \\ Beijing, China \\ e-mail: netbaimingliu@sina.com
}

\author{
Weiwei \\ Beijing University of Agriculture \\ Beijing, China \\ e-mail: 281501616@qq.com
}

\begin{abstract}
This article has analyzed the insufficiency of the traditional teaching administration system, proposed that through inserts the way of regulation storeroom to realize in the procedure active database-based the teaching administration system, through the practical research showed that this system can provide the real-time, all-round service on own initiative, and active service mechanism is highly effective, feasible

In this paper, it studies one of the error check cont rols of the communications of computer's network . Cyclic Redundancy Check( CRC ) . It introduces the principle of CRC, the algorithms of CRC, the algorithms analysis of CRC, the program of CRC and the functions \& features of CRC. The algorithms of CRC need not to design circuit of hardware in addition. It improves the speed of the communications of computer' s network an d checks the message correctly.
\end{abstract}

Keywords- Cyclic Redun dancy Check ( CRC ) ; Algorithm of CRC; Comput er;s Netw ork; Error Check Cont rol

\section{INTRODUCTION}

The computer network is distributed in different geographic locations, The entity (user applications, file transfer packet, the database management system,E-mail devices, terminals, etc.), data communication lines linking Lee Communication protocols to communicate, to realize the sharing of the resources of the entire system. Exchange of information in the network, the entity must be established between the data communication lines to It efficient and accurate transmission of information. In practical applications, both A remote data communication line or a local data communication line, are not Be subject to a variety of the influence of interference, so that the information received by the receiving end Issued inconsistent with the sender that the information received by the receiving end production The error. Metric data communication line transmission channel bit error rate (Pc)

The accuracy of the interest rate technical support for the school educational administration.

Then

Pc = Error received symbol $/$ number of received symbols

In order to reduce the error rate of transmission of data communication lines, usually there are changes.Good transmission quality of data communication lines and error detection control of two sides. Method. Improve data communication line transmission quality is the introduction of a new set of exchange Prepared, the new data communication line, the new technology. However, this method.Due to the economic and technical constraints, in order to achieve a very low false Bit rate, spend consideration but with little success. In fact all of the computer Network communication system using error detection and control, and to acknowledge that the data communication Line transmission information error conditions, to effectively detect error conditions, and thus Line corrected, in order to improve the quality of transmission of the data communication lines. Error detection.Measured a lot of control, this article only describes cyclical redundancy check CRC(Cyclic Redundancy Check) algorithm, it is in the data communication line Well check the transmission of information.

\section{CRC ALGORITHM PRINCIPLE}

The exchange of information between the entities of the computer network, the first Extended packets transmitted is divided into a smaller, equal length data segments, In front of each data segment with the first portion (Head), the checksum is added to the end Code, constitute a data packet (Data Packet). In a data communication line Road transmission is a data packet, the receiving side computer from the data packet was To the needs of the various control information and the transmission of useful information, so that the Transmission error detection control has become a management work. The packet format is shown in Figure 1. , $\mathrm{SOH}$ header usually contains Important control signals for synchronization information, the source station address and destination address, etc. Interest; serial packets into data packets the number of groups, i.e., several groups of data packets; The length is the number of bytes of the packet data; data is useful for transmission Information; the packet checksum is calculated by the CRC algorithm The data CRC code.

\begin{tabular}{|l|l|l|l|l|}
\hline $\mathrm{SOH}$ & $\begin{array}{l}\text { Serial } \\
\text { number }\end{array}$ & .Length & Data & checksum \\
\hline
\end{tabular}

Figure 1 packet format

The message packet transport protocol, the sender computer using CRC algorithm calculated from the data to be sent out of the CRC code (two bytes), and CRC code check code is added to the end of the packet. After receiving 
side computer receives a data packet with a CRC algorithm performs the same calculation, and the calculated CRC code to be compared with the received CRC code. If the two sets of CRC code value is equal, followed by the transmission of the next data packet; if not equal, indicating that the transmission error, the transmitting side computer through the ARQ (Automatic Repeat re Quest) approach requires the packet retransmission. The package after the CRC checksum transmission of data is very reliable, and has been adopted by most asynchronous communication software. CRC checksum technique to process the data in a data packet as a bit string, its checksum data from the data packet of the first byte of a start to the end of the last one of the last byte.

CRC algorithm is commonly used in the computer network communication error detection control method, and its main purpose is to reduce the transmission error of the data communication lines.The main idea of CRC algorithms is: while increasing the CRC code at the transmitting end transmitting data between the data and the CRC code to establish a mathematical relationship (i.e., the encoding process); When these data and the CRC code sent to the receiving end,by the mathematical relationship between the detection data and the CRC code of the receiving side (decoding process) is correct, and if correct, it shows the error in the transmission process, usually by the weight of the transmitting side came correcting errors until the transfer of the correct date.Be seen how effective error detection is the key to this approach

\section{CRC ALGORITHM VALIDATION RULES}

The CRC algorithm validation rules is to make the calibration data for a generator polynomial In addition, if in addition to have the best that the code is correct; indivisible remainder will indicate the dislocation location.Any set of calibration data is likely indivisible, will produce a remainder, if so check data minus the remainder bound for the generator polynomial divisible,But,Subtraction operation may need to borrow computing, it is difficult to achieve with a simple method of assembling coding. Therefore a standard modulo 2, i.e. through the die 2 subtracter realize the modulo-2 divider, and a modulo 2 adder the resultant remainder splice is formed in the back of the check data, a divisible by the parity data. Of course, the use of modulo 2 division, the choice of the generator polynomial is conditional. The CRC algorithm modulo 2 is a four bit up / down based computing, does not consider the carry and borrow, and to die four fixed-point decimal arithmetic are two different concepts. Therefore, the modulo-2 addition / subtraction that bit up / down, also is the exclusive OR operation available XOR gates.

By check data is a set of information code may be the figures representing the numerical size, it may be character encoding or other nature of the code, but it shows in the form of a binary ( 0,1 code) in the transmission.Modulo- 2 addition to temporarily check data as numbers, described by the polynomial.Defined by the parity data (dividend) M (x); convention of the generator polynomial (divisor) of the $G$ (x), is used to produce a remainder; generated by the remainder $\mathrm{R}(\mathrm{x})$, which corresponds to thewith redundant parity bits.

\section{A. Generating polynomial of the selected}

Not any polynomial can be used as a generator polynomial, error detection and error correction requirements, generating polynomials should be able to meet the following requirements: an error occurred should remainder 0 ; differentThe bit error occurs should the remainder; should meet the cyclical nature of the remainder.Recommended by the International Telegraph and Telephone Consultative Committee (CCITT) 16-bit (two bytes) CRC code generator polynomial $\mathrm{G}(\mathrm{X})=\mathrm{X}^{16}+\mathrm{X}^{12}+\mathrm{X}^{5}+1$ where $\mathrm{X}$ is $\mathrm{a}$ value of 2

CRC algorithm can detect all of the parity data of one or two errors; all having an odd number of bit errors; all burst errors of less than 16; burst error of more than 16 bits detected probability99.9\%. This level of error detection is the computer network communication information required for the transmission.

\section{B. Encoding method}

Will be left $r$ bits of $k$ bits of effective information of the verification data $\mathrm{M}(\mathrm{x})$ to give $\mathrm{M}(\mathrm{X}) . \mathrm{X}^{r}$ The purpose of doing so is to empty out $r$ bits, in order to assemble the future determined r-bit remainder.Be $r$ bits of the $k$ bits of effective information of the verification data $M$ (x) left, $\mathrm{M}(\mathrm{x}) \cdot \mathrm{x}^{\mathrm{r}}$. The purpose of doing so is to empty out $\mathrm{r}$ bits, in order to assemble the future determined $r$ bit remainder.

Select an r+1-bit generator polynomial, $\mathrm{G}(\mathrm{x})=\mathrm{X}^{16}+\mathrm{X}^{12}+\mathrm{X}^{5}+1$, Modulo 2 division of the $\mathrm{M}(\mathrm{x}) . \mathrm{x}^{\mathrm{r}}$.

$$
\frac{\mathrm{M}(\mathrm{x}) \cdot \mathrm{x}^{\mathrm{r}}}{\mathrm{G}(\mathrm{x})}=Q(\mathrm{x})+\frac{\mathrm{R}(\mathrm{x})}{\mathrm{G}(\mathrm{x})}
$$

To produce the r-bit remainder, so the generator polynomial $\mathrm{G}(\mathrm{x})$ is the $\mathrm{r}+1$ bit

Left shift $r$ bits to be programmed valid information and the remainder $\mathrm{R}(\mathrm{x})$ modulo 2 plus (minus), that splicing of cyclic checksum

$$
\mathrm{M}(\mathrm{x}) \cdot \mathrm{x}^{\mathrm{r}}+\mathrm{R}(\mathrm{x})=\mathrm{Q}(\mathrm{x}) \cdot \mathrm{G}(\mathrm{x})
$$

Bitwise modulo-2 addition with mold subtraction results are consistent, So

$$
M(x) \cdot x^{r}-R(x)=M(x) \cdot x^{r}+R(x) T
$$

he end of the $\mathrm{r} M(\mathrm{X}) \mathrm{X}^{r} \mathrm{R}$ bit is a 0 , so the remainder $\mathrm{R}(\mathrm{x})$ of the add / subtract is actually M (x) R (x) splicing.Spliced into the checksum must be divisible agreed $\mathrm{G}(\mathrm{x})$. 


\section{Decoding and error correction}

Cyclic check code generator polynomial G (x) using the convention will be notified of the removal. If the code word is correct, the remainder 0 ; If a bit error, the remainder is not 0 , and the different bit error remainder different, unique correspondence relationship between the serial number of the code and the syndrome of the remainder, can be through the lookup table to determine.

\section{CRC ALGORITHM ANALYSIS}

CRC algorithm parameters of a 512-byte table, can be found in the parameter list parameter values for each byte. CRC algorithm is designed, pre-opened to store the 512-byte buffer to store the parameter table, so that the different variables to store the CRC code and the intermediate result. Sequentially taken every byte of the packet data from the parameter table found its parameter value 1 . This word Section XOR on a byte low 8 verified, and then, and then XOR the byte to identify high eight results 1 to 1 to send high 8 bytes. The low eight bytes identify the value XOR on 8 verified, and then the high of one byte worth results to the results of 2 Get low 8 bytes. On a one-byte checksum of the low eight, to find the parameter values from the parameter table. 1 results and parameter values after the exclusive-or, as the byte checksum. Until the packet data checksum finished, get a CRC code.

CRC algorithm for loop sequentially taken every byte of the packet data, and then calculated for each byte, the time complexity of the CRC algorithm degree of $O(n)$, greatly improved the speed of the parity.Space complexity of the variables stored in the parameter list of the 512-byte buffer and store intermediate results.Relative to the other algorithms, CRC algorithm takes up more storage space, but with the improvement of computer performance, increasing memory space for time is worth advocating.

\section{CRC ALGORITHM PROGRAMMING}

CRC algorithm program design is obtained according to the CRC algorithm analysis, the user can be prepared in accordance with the computer network communication the main program, the CRC () subroutine is called to calculate a CRC code of the data packet, and then the CRC code is added to the data packet as a correctionThe check code transmitted to the receiving side computer.After receiving side computer receives a data packet with a CRC algorithm performs the same calculation, and the calculated CRC code to be compared with the received CRC code, thereby to determine whether the received data packet is correct.

The CRC algorithm parameter table (512 bytes) omitted expressed as follows:

Const byte crcchar $[512]=\{0 x 00,0 x 00,0 x 89,0 \times 10$, , 0xe0,0x78, 0x f0\}

CRC algorithm source code as follows:

\# define COMBU F 1024 / / Buffer Size

\# define ETX ( byte) 0x 3 / / Message terminator byte $\operatorname{crc} 0=0, \operatorname{crc} 1=0 ; / /$ Byte variable

word $\operatorname{crc}$ ( byt e * cbuf) / / Calculation of the CRC code of the data packet, and returns the CRC code $\{$ word $\mathrm{i}$; for $(\operatorname{crc} 0=$ $\operatorname{crc} 1=0, \mathrm{i}=1$; $\operatorname{cbuf}[\mathrm{i}]$ ! = ETX \&\& $\mathrm{i}<$ COMBUF; $\mathrm{i}+$ + ) crcby te( cbuf[i] );

if $(\mathrm{i}+4>=$ COMBUF $)$ return 0 ; crcbyte ( cbuf $[\mathrm{i}])$;

$\operatorname{cbuf}(++\mathrm{i})=\operatorname{crc} 0 ; \operatorname{cbuf}(++\mathrm{i})=\operatorname{crc} 1 ; \operatorname{cbuf}(++\mathrm{i})=\operatorname{crc} 0$; $\operatorname{cbuf}(++\mathrm{i})=\operatorname{crc} 1 ; \operatorname{cbuf}(++\mathrm{i})=\operatorname{crc} 0 ; \operatorname{cbuf}(++\mathrm{i})=\operatorname{crc} 1$; return $++\mathrm{i}$; \}

vo id crcby te( byte crc30) / / Calculating a byte CRC code and is returned as the byte Checksum

\{ byte crc10, crc 11 , crc 20 , crc 21 ; word crclen; crclen $=($ word $)$ crc30; crclen $=($ crclen $\& 0 x$ ff $)<<1$;

$\operatorname{crc} 10=\operatorname{crcchar}[$

crclen+ + ] ;

$\operatorname{crc} 11=\operatorname{crcchar}[\operatorname{crclen}++] ; \operatorname{crc} 11=\operatorname{crc} 30^{\wedge} \operatorname{crc} 0^{\wedge} \operatorname{crc} 11$;

$\operatorname{crc} 10=\operatorname{crc} 1 \wedge$

crc10;

crclen $=($ word $)$ crc0; crclen $=($ crclen $\& 0 x f f)<<1$; crc20 $=$ crcchar

[ crclen+ + ] ;

$\operatorname{crc} 21=\operatorname{crcchar}[\operatorname{crclen}++] ; \operatorname{crc} 0=\operatorname{drd} \wedge^{\wedge} \operatorname{crc} 20 ; \operatorname{crc} 1=$ $\left.\operatorname{crc} 11^{\wedge} \operatorname{crc} 21 ;\right\}$

\section{CONCLUSION}

CRC algorithm implemented in software, its main advantage is saving hardware, no additional design the hardware circuit, check the speed is very fast.Parameter tables and applications into memory when the need to verify the packet CRC algorithm calculation, you can get the checksum of the packet.CRC algorithm to improve the communication speed, preferably overcome the contradiction of the asynchronous transmission, the check and the transmission time is very match, and is commonly used in the computer network communication error detection control method.

\section{REFERENCES}

[1] Ying-Shing CHAN. Formation of brain map for spatial perception. Proceedings of the 8th Biennial Conference of the Chinese Society for Neuroscience,2009, pp. 102-103

[2] D.K.Y.Shum. Expression of chondroitin sulfotransferases in cranial motor neurons in the embryonic rat hindbrain. Proceedings of the 8th Biennial Conference of the Chinese Society for Neuroscience,2009 , pp. 12-21

[3] Ying-Shing CHAN. Contribution of the thalamic reticular nucleus to the synchronization of spindle oscillation in the thalamocortical network. Proceedings of the 8th Biennial Conference of the Chinese Society for Neuroscience. 2009, pp. 202-210

[4] Darrell Li,Paul Wen,D.K.Luk. Application of diffusion tensor imaging fiber tracking in cervical spondylotic myelopathy. Proceedings of the 8th Biennial Conference of the Chinese Society for Neuroscience, 2009, pp. 56-61

[5] Amy Cheuk-Yin LO,Chung-Man YEUNG,Suk-Yee LI,Raymond Chuen-Chung CHANG,Kwok-Fai SO,David WONG. Neuroprotective effects of Lycium barbarum polysaccharides on acute ischemic stroke injury.Proceedings of the 8th Biennial Conference of the Chinese Society for Neuroscience. 2009 , pp. 78-82 